\title{
Dynamic Data Path Prediction in Network Virtual Environment
}

\author{
Sun-Hee Song, Seung-Moon Jeong, Gi-Taek Hur ${ }^{1}$, and Sang-Dong $\mathrm{Ra}^{2}$ \\ Digital Contents Cooperative Research Center Dongshin University, \\ ${ }^{1}$ Dept. of Digital Contents, Dongshin University \\ ${ }^{2}$ Dept. of Computer Engineering, Chosun University \\ shsong@dsu.ac.kr
}

\begin{abstract}
This research studies real time interaction and dynamic data shared through 3D scenes in virtual network environments. In a distributed virtual environment of client-server structure, consistency is maintained by the static information exchange; as jerks occur by packet delay when updating messages of dynamic data exchanges are broadcasted disorderly, the network bottleneck is reduced by predicting the movement path by using the Dead-reckoning algorithm. The shared dynamic data of the 3D virtual environment is implementation using the VRML EAI.
\end{abstract}

Keywords: net-VE, Dead-reckoning, Consistency.

\section{Introduction}

Net-VE(Network Virtual Environment)[1][2] is a system that connection the distributed network to the virtual reality technology and offers 3D space to cooperate distributed multi-users interaction through realtime networking. Consistency at a distributed virtual environment[3] of the client-server structure is continued by continuous exchange of static information among distributed clients. The cycles transfer of static information brings traffic overhead of network. The precise way for network users to know the others' static is to transfer the packet by hand shaking for each frame, which takes a overload of the synchronization and decrease the velocity.

Based on the roles by which the dynamic data of the distributed multi-users is processed through the multi-casting communication via the client-server and the peerpeer server, the network system in this study is composed of the message server and the application server and distribution servicing loads by allocating realtime data to the dynamic data server and non-realtime data to the static data server. When a new client is connecter to a 3D scene of the network virtual space, it interpolates the prior location with the Dead-reckoning[4] path prediction algorithm of DIS(Distributed Interactive Simulation) to continue consistency and presentation the dynamic data sharing scene of the 3D virtual space. 


\section{Dynamic Data Path Prediction}

\subsection{Path Prediction Using Dead-Reckoning Algorithm}

When you know the current location $x(t)$, the location at the time change $t+\Delta t$ after movement for a cycle interval from the time $t$ at an average velocity can be calculated as in expression (1). Based on the location of the shared object in expression (1), the object location at the current time can be estimated. The previous location is interpolated if the error is over the predetermined threshold after reviewing the error between the estimated and the actual static values.

$$
\begin{gathered}
x(t+\Delta t)=x(t)+\dot{x} \Delta t \quad \dot{x}=V \cos \psi \\
y(t+\Delta t)=y(t)+\dot{y} \Delta t \quad \dot{y}=V \sin \psi \\
V: \text { average velocity at the time }[t, t+\Delta t] \\
\psi: \text { average direction angle at the time }[t, t+\Delta t]
\end{gathered}
$$

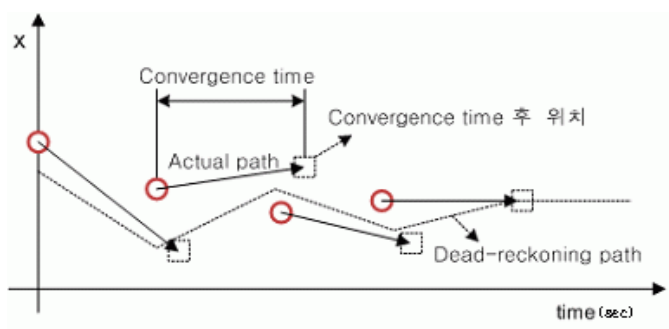

Fig. 1. Dead-reckoning Convergence

Fig. 1 shows the Dead-reckoning convergence process. We can get more precise estimates by increasing the estimate function interval of expression (2) but it results in more composite calculation. Therefore, we use $2^{\text {nd }}$ level functions such as $1^{\text {st }}$ differentials or $2^{\text {nd }}$ differentials. We adjusts the threshold of the Dead-reckoning convergence number and control the static information transfer rate. The client that receives the discreteness dynamic data static information creates a continually shared static using the shared static location convergence expression (2)

$$
x(t)=x\left(t_{0}\right)+\left(t-t_{0}\right) \frac{d x(t)}{d t} \mathrm{I}_{t=t_{0}}+\frac{\left(t-t_{0}\right)^{2}}{2} \frac{d^{2} x(t)}{d t^{2}} \mathrm{|}_{t=t_{0}}+\cdots
$$

Fig. 2. shows the measurement of the actual location, the convergence location and the estimated location error when the path from the initial value of the dynamic data by the Dead-reckoning convergence width, $(x, y, \theta)=\left(1.5,1.8,70.0^{\circ}\right)$, to the value, $\left(x_{n}, y_{n}, \theta_{n}\right)=\left(4.62,5.64,70.0^{\circ}\right)$, is set to the velocity 2.4 , the acceleration 0 , the time stamp 2.0, and the DR Interval $0.75,0.50,0.10,0.05$. When the actual location in Table 1 and the location prediction error by convergence width are measured at the point $\left(x_{n}, y_{n}, \theta_{n}\right)=\left(4.62,5.64,70.0^{\circ}\right)$, the estimated error rate gets smaller and it becomes possible to predict the location which is closer to the actual path when the 
Dead-reckoning convergence width is adjusted between 0.05 and 0.5 , as shown in Fig. 2. As the location prediction interpolation error is 0 or -0.01 in (b) DR Interval = 0.10 and (c) DR Interval $=0.05$, the dynamic data movement is not sensed at the client rendering. Although realtime rendering is more possible as the consistency is higher, it is possible to send the location change information of the shared object to the other clients and continue an proper transfer rate when in 0.10 , because the server function and frequent updates cause network broadband width delays.

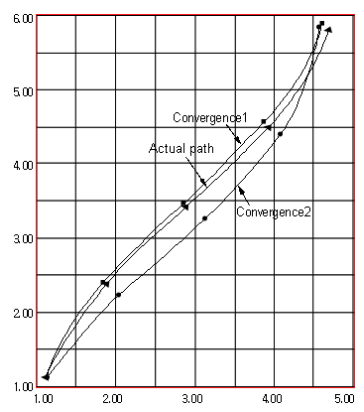

(a) DR Interval $=0.50$

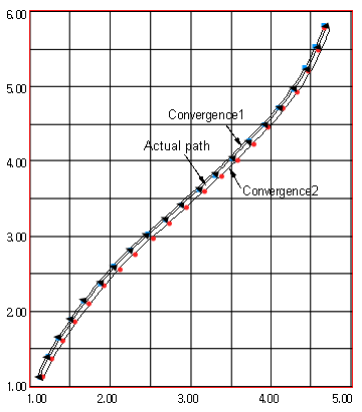

(b) DR Interval $=0.10$

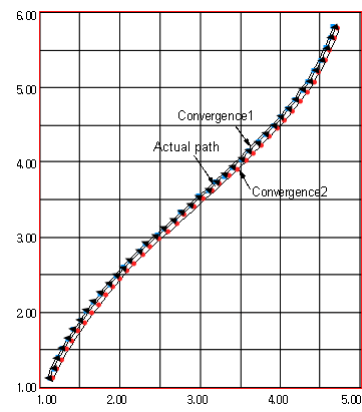

(c) DR Interval $=0.05$

Fig. 2. Position Prediction of Dead-reckoning Convergence

The location interpolation includes check of the error between the estimated and the real values and interpolation of the previous location when the error is over the predetermined threshold of the Dead-reckoning convergence. If the threshold is big, the average transfer rate of static information is low, even though the error of the shared static gets bigger. If the threshold is small, the average transfer rate and broadband width get heighten even though the error of the shared static gets smaller. $P_{t 0}$ and $V^{r}$ mean the ESPDU location and velocity, respectively. expression (3)is for interpolating the previous location of the entity using the initial location value $t_{0}$ by the time stamp velocity at the linear block $d_{n}$ and the location estimate $t_{1}$.

$$
P_{t 1}=P_{t 0}+V^{r}\left(t_{1}-t_{0}\right)
$$

\section{Conclusion}

The dynamic data whose path was predicted by a Dead-reckoning algorithm interpolates the previous location with an interpolation node(Interpolation), transfers the shared object static information, and continues consistency with other clients.

At the network 3D virtual space, the movement path was predicted using the Deadreckoning algorithm at the client buffer because the congested broadcast by interaction and static information caused network delay and jerks. The error between the estimated and the actual static values, which is more than the threshold based on 
the shared object location, required interpolation of the prior location using the Deadreckoning estimate function and multicasting of the ESPDU packet of the DIS.

Fig. 3 is the 3D scene with the output through the client rendering engine at the network virtual space. The actual path of the dynamic data agent_A is 'Actual Path', and as the Dead-reckoning estimate location path is a 'DR path' and the dynamic data moves suddenly when the user who received the shared static updates the information, it does not change right away to a client cache value, but moves to the 'Interpolation path' by the convergence interval.

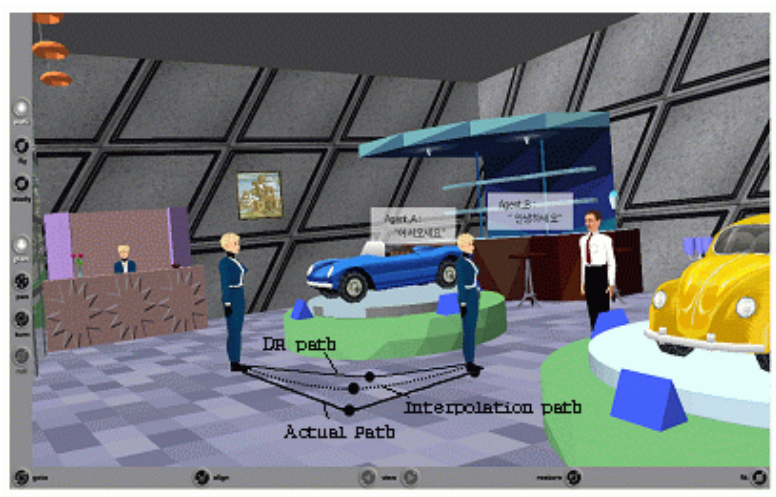

Fig. 3. Dead-reckoning Apply of 3D Graphics Scene

\section{References}

[1] Singhal, S. and Zyda, M., 1999. Networked Virtual Environments: Design and Implementation, ACM Press [ISBN 0-201-32557-8].

[2] Bouras, C., Triantafillou, V. and Tsiatsos, T., 2001. Aspects of collaborative learning environment using distributed virtual environments In Proceedings of ED-MEDIA, Tampere, Finland, June25-30 pp.173-178

[3] Bouras,C., Psaltoulis, D., Psaroudis, C. and Tsiatsos, T., 2003. Multi user layer in the EVE distributed virtual reality platform In Proceedings of Fifth International Workshop on Multimedia Networks Systems and Applications (MNSA 2003) Providence, Rhode Island, USA, May 19-22 pp.602-607.

[4] W. Cai, F.B.S. Lee, L. Chen, An auto-adaptive Dead-reckoning algorithm for distributed interactive simulation, in: Proceedings of the Thirteenth Workshop on Parallel and Distributed Simulation, 1999, pp.82-89. 